\title{
Beam-charge azimuthal asymmetry and deeply virtual Compton scattering
}

A. Airapetian, ${ }^{17}$ N. Akopov,${ }^{29}$ Z. Akopov, ${ }^{29}$ M. Amarian,${ }^{8,29}$ A. Andrus, ${ }^{16}$ E. C. Aschenauer, ${ }^{8}$ W. Augustyniak,${ }^{28}$ R. Avakian, ${ }^{29}$ A. Avetissian, ${ }^{29}$ E. Avetissian, ${ }^{12}$ P. Bailey, ${ }^{16}$ D. Balin, ${ }^{20}$ M. Beckmann, ${ }^{7}$ S. Belostotski, ${ }^{20}$ N. Bianchi, ${ }^{12}$ H. P. Blok, ${ }^{19,27}$ H. Böttcher, ${ }^{8}$ A. Borissov ${ }^{15}$ A. Borysenko, ${ }^{12}$ M. Bouwhuis,${ }^{16}$ A. Brüll, ${ }^{30}$ V. Bryzgalov,${ }^{21}$ M. Capiluppi,${ }^{11}$ G. P. Capitani, ${ }^{12}$ T. Chen, ${ }^{4}$ G. Ciullo, ${ }^{11}$ M. Contalbrigo, ${ }^{11}$ P. F. Dalpiaz, ${ }^{11}$ W. Deconinck, ${ }^{17}$ R. De Leo, ${ }^{3}$ M. Demey, ${ }^{19}$ L. De Nardo, ${ }^{1}$ E. De Sanctis, ${ }^{12}$ E. Devitsin, ${ }^{18}$ P. Di Nezza, ${ }^{12}$ J. Dreschler, ${ }^{19}$ M. Düren, ${ }^{14}$ M. Ehrenfried,${ }^{10}$

A. Elalaoui-Moulay, ${ }^{2}$ G. Elbakian, ${ }^{29}$ F. Ellinghaus, ${ }^{6}$ U. Elschenbroich, ${ }^{13}$ R. Fabbri, ${ }^{19}$ A. Fantoni, ${ }^{12}$ L. Felawka, ${ }^{25}$ S. Frullani, ${ }^{23}$ A. Funel, ${ }^{12}$ G. Gapienko, ${ }^{21}$ V. Gapienko, ${ }^{21}$ F. Garibaldi, ${ }^{23}$ K. Garrow, ${ }^{25}$ D. Gaskell, ${ }^{6}$ G. Gavrilov, ${ }^{7,20,25}$ V. Gharibyan,$^{29}$ O. Grebeniouk,${ }^{20}$ I. M. Gregor,${ }^{8}$ C. Hadjidakis, ${ }^{12}$ K. Hafidi, ${ }^{2}$ M. Hartig, ${ }^{14}$ D. Hasch, ${ }^{12}$

W. H. A. Hesselink, ${ }^{19,27}$ A. Hillenbrand,,${ }^{10}$ M. Hoek, ${ }^{14}$ Y. Holler, ${ }^{7}$ B. Hommez, ${ }^{13}$ I. Hristova, ${ }^{8}$ G. Iarygin, ${ }^{9}$ A. Ivanilov, ${ }^{21}$ A. Izotov, ${ }^{20}$ H. E. Jackson, ${ }^{2}$ A. Jgoun,${ }^{20}$ R. Kaiser, ${ }^{15}$ E. Kinney, ${ }^{6}$ A. Kisselev, ${ }^{6,20}$ T. Kobayashi, ${ }^{26}$ M. Kopytin, ${ }^{8}$ V. Korotkov, ${ }^{21}$ V. Kozlov,${ }^{18}$ B. Krauss,${ }^{10}$ V. G. Krivokhijine, ${ }^{9}$ L. Lagamba,${ }^{3}$ L. Lapikás, ${ }^{19}$ A. Laziev, ${ }^{19,27}$ P. Lenisa, ${ }^{11}$ P. Liebing, ${ }^{8}$ L. A. Linden-Levy, ${ }^{16}$ W. Lorenzon, ${ }^{17}$ H. Lu, ${ }^{5}$ J. Lu, ${ }^{25}$ S. Lu, ${ }^{14}$ B.-Q. Ma, ${ }^{4}$ B. Maiheu, ${ }^{13}$ N. C. R. Makins, ${ }^{16}$ Y. Mao, ${ }^{4}$ B. Marianski, ${ }^{28}$ H. Marukyan,${ }^{29}$ F. Masoli, ${ }^{11}$ V. Mexner, ${ }^{19}$ N. Meyners, ${ }^{7}$ T. Michler, ${ }^{10}$ O. Mikloukho, ${ }^{20}$ C. A. Miller, ${ }^{1,25}$ Y. Miyachi, ${ }^{26}$ V. Muccifora, ${ }^{12}$ M. Murray, ${ }^{15}$ A. Nagaitsev, ${ }^{9}$ E. Nappi,${ }^{3}$ Y. Naryshkin, ${ }^{20}$ M. Negodaev, ${ }^{8}$ W.-D. Nowak, ${ }^{8}$ K. Oganessyan,,${ }^{7,12}$ H. Ohsuga, ${ }^{26}$ A. Osborne, ${ }^{15}$ N. Pickert, ${ }^{10}$ D. H. Potterveld, ${ }^{2}$ M. Raithel, ${ }^{10}$ D. Reggiani, ${ }^{10}$ P.E. Reimer, ${ }^{2}$ A. Reischl, ${ }^{19}$ A. R. Reolon, ${ }^{12}$ C. Riedl,${ }^{10}$ K. Rith, ${ }^{10}$ G. Rosner, ${ }^{15}$ A. Rostomyan, ${ }^{29}$ L. Rubacek, ${ }^{14}$ J. Rubin, ${ }^{16}$ D. Ryckbosch, ${ }^{13}$ Y. Salomatin, ${ }^{21}$ I. Sanjiev, ${ }^{20}$ I. Savin, ${ }^{9}$ A. Schäfer, ${ }^{22}$ G. Schnell, ${ }^{26}$ K. P. Schüler, ${ }^{7}$ J. Seele, ${ }^{6}$ R. Seidl, ${ }^{10}$ B. Seitz, ${ }^{14}$ R. Shanidze, ${ }^{10}$ C. Shearer, ${ }^{15}$ T.-A. Shibata, ${ }^{26}$ V. Shutov, ${ }^{9}$ K. Sinram, ${ }^{7}$ W. Sommer, ${ }^{14}$ M. Stancari, ${ }^{11}$ M. Statera, ${ }^{11}$ E. Steffens, ${ }^{10}$ J. J. M. Steijger, ${ }^{19}$ H. Stenzel, ${ }^{14}$ J. Stewart, ${ }^{8}$ F. Stinzing, ${ }^{10}$ P. Tait, ${ }^{10} \mathrm{H}$. Tanaka, ${ }^{26} \mathrm{~S}$. Taroian, ${ }^{29}$ B. Tchuiko, ${ }^{21}$ A. Terkulov ${ }^{18}$ A. Trzcinski, ${ }^{28}$ M. Tytgat, ${ }^{13}$ A. Vandenbroucke, ${ }^{13}$ P. B. van der Nat, ${ }^{19}$ G. van der Steenhoven, ${ }^{19}$ Y. van Haarlem, ${ }^{13}$ V. Vikhrov, ${ }^{20}$ M. G. Vincter, ${ }^{1}$ C. Vogel,${ }^{10}$ J. Volmer, ${ }^{8}$ S. Wang, ${ }^{4}$ J. Wendland,${ }^{24,25}$ Y. Ye, ${ }^{5}$ Z. Ye ${ }^{7}$ S. Yen, ${ }^{25}$ B. Zihlmann, ${ }^{13}$ and P. Zupranski ${ }^{28}$

\section{(HERMES Collaboration)}

\footnotetext{
${ }^{1}$ Department of Physics, University of Alberta, Edmonton, Alberta T6G 2J1, Canada

${ }^{2}$ Physics Division, Argonne National Laboratory, Argonne, Illinois 60439-4843, USA

${ }^{3}$ Istituto Nazionale di Fisica Nucleare, Sezione di Bari, 70124 Bari, Italy

${ }^{4}$ School of Physics, Peking University, Beijing 100871, China

${ }^{5}$ Department of Modern Physics, University of Science and Technology of China, Hefei, Anhui 230026, China

${ }^{6}$ Nuclear Physics Laboratory, University of Colorado, Boulder, Colorado 80309-0390, USA

${ }^{7}$ DESY, 22603 Hamburg, Germany

${ }^{8}$ DESY, 15738 Zeuthen, Germany

${ }^{9}$ Joint Institute for Nuclear Research, 141980 Dubna, Russia

${ }^{10}$ Physikalisches Institut, Universität Erlangen-Nürnberg, 91058 Erlangen, Germany

${ }^{11}$ Istituto Nazionale di Fisica Nucleare, Sezione di Ferrara and Dipartimento di Fisica, Università di Ferrara, 44100 Ferrara, Italy

${ }^{12}$ Istituto Nazionale di Fisica Nucleare, Laboratori Nazionali di Frascati, 00044 Frascati, Italy

${ }^{13}$ Department of Subatomic and Radiation Physics, University of Gent, 9000 Gent, Belgium

${ }^{14}$ Physikalisches Institut, Universität Gießen, 35392 Gießen, Germany

${ }^{15}$ Department of Physics and Astronomy, University of Glasgow, Glasgow G12 8QQ, United Kingdom

${ }^{16}$ Department of Physics, University of Illinois, Urbana, Illinois 61801-3080, USA

${ }^{17}$ Randall Laboratory of Physics, University of Michigan, Ann Arbor, Michigan 48109-1040, USA

${ }^{18}$ Lebedev Physical Institute, 117924 Moscow, Russia

${ }^{19}$ Nationaal Instituut voor Kernfysica en Hoge-Energiefysica (NIKHEF), 1009 DB Amsterdam, The Netherlands

${ }^{20}$ Petersburg Nuclear Physics Institute, St. Petersburg, Gatchina, 188350 Russia

${ }^{21}$ Institute for High Energy Physics, Protvino, Moscow region, 142281 Russia

${ }^{22}$ Institut für Theoretische Physik, Universität Regensburg, 93040 Regensburg, Germany

${ }^{23}$ Istituto Nazionale di Fisica Nucleare, Sezione Roma 1, Gruppo Sanità and Physics Laboratory, Istituto Superiore di Sanità, 00161 Roma, Italy

${ }^{24}$ Department of Physics, Simon Fraser University, Burnaby, British Columbia V5A 1S6, Canada

${ }^{25}$ TRIUMF, Vancouver, British Columbia V6T 2A3, Canada

${ }^{26}$ Department of Physics, Tokyo Institute of Technology, Tokyo 152, Japan

${ }^{27}$ Department of Physics and Astronomy, Vrije Universiteit, 1081 HV Amsterdam, The Netherlands

${ }^{28}$ Andrzej Soltan Institute for Nuclear Studies, 00-689 Warsaw, Poland
} 
${ }^{29}$ Yerevan Physics Institute, 375036 Yerevan, Armenia

${ }^{30} 36$ Mizzen Circle, Hampton, Virginia 23664, USA

(Received 25 May 2006; published 26 January 2007)

The first observation of an azimuthal cross section asymmetry with respect to the charge of the incoming lepton beam is reported from a study of hard exclusive electroproduction of real photons. The data have been accumulated by the HERMES experiment at DESY, in which the HERA 27.6 GeV electron or positron beam scattered off an unpolarized hydrogen gas target. The observed asymmetry is attributed to the interference between the Bethe-Heitler process and the deeply virtual Compton scattering (DVCS) process. The interference term is sensitive to DVCS amplitudes, which provide the most direct access to generalized parton distributions.

DOI: 10.1103/PhysRevD.75.011103

PACS numbers: 13.60.Fz, 14.20.Dh, 24.85.+p

The partonic structure of the nucleon has been traditionally described in terms of parton distribution functions (PDFs), which appear in the interpretation of, e.g., inclusive deeply inelastic scattering (DIS). More recently, PDFs have been subsumed within generalized parton distributions (GPDs) [1-3], which relate directly to hard exclusive processes that involve at least one additional hard vertex, yet leave the target nucleon intact. The ordinary PDFs and nucleon elastic form factors appear as kinematic limits and moments of GPDs, respectively. Strong interest in the formalism of GPDs has emerged after GPDs were found to offer the first possibility to reveal the total angular momentum carried by the quarks in the nucleon [2]. More recent discussions focus on the potential of GPDs as a three-dimensional representation of hadrons at the parton level [4-8].

Among all practical probes, the deeply virtual Compton scattering (DVCS) process, i.e., the hard exclusive leptoproduction of a real photon $(e p \rightarrow e p \gamma)$, appears to provide the theoretically cleanest access to GPDs. Direct access to the DVCS amplitudes is provided by the interference between the DVCS and Bethe-Heitler $(\mathrm{BH})$ processes, in which the photon is radiated from a parton and from the lepton, respectively. Since these processes have an identical final state, the squared photon production amplitude is given by

$$
|\tau|^{2}=\left|\tau_{\mathrm{BH}}\right|^{2}+\left|\tau_{\mathrm{DVCS}}\right|^{2}+\underbrace{\tau_{\mathrm{DVCS}} \tau_{\mathrm{BH}}^{*}+\tau_{\mathrm{DVCS}}^{*} \tau_{\mathrm{BH}}}_{I},
$$

where $I$ denotes the interference term. It introduces a dependence on the beam charge, which is a rare phenomenon normally confined to processes involving the weak interaction. The $\mathrm{BH}$ amplitude $\left(\tau_{\mathrm{BH}}\right)$ is precisely calculable from measured elastic form factors. The cross section depends on the Bjorken scaling variable $x_{B}$, the squared virtual-photon four-momentum $-Q^{2}$, and the squared four-momentum transfer $t$ to the target. This interpretation of the virtual-photon kinematics does not apply to the BH process. In addition, the cross section depends on the azimuthal angle $\phi \in[-\pi, \pi]$, defined as the angle between the plane containing the incoming and outgoing lepton trajectories and the plane correspondingly defined by the virtual and real photon [9].
For an unpolarized proton target, and $-t \ll Q^{2}$, the interference term is given by [10]

$$
I \propto-C\left[a \cos \phi \operatorname{Re} \tilde{\mathcal{M}}^{1,1}-b P_{l} \sin \phi \operatorname{Im} \tilde{\mathcal{M}}^{1,1}\right],
$$

where the lepton beam has longitudinal polarization $P_{l}$ and charge $C= \pm 1$, and $a$ and $b$ are functions of the ratio of longitudinal to transverse virtual-photon flux. A polarization-independent constant term, higher harmonics $(\cos 2 \phi, \cos 3 \phi, \sin 2 \phi)$, as well as a $\cos \phi$ dependence in the prefactor, have been neglected since they are suppressed by at least $\mathcal{O}(1 / Q)$ or $\mathcal{O}\left(\alpha_{s}\right)$. Here $\alpha_{s}$ is the strong coupling constant. The squared BH and DVCS amplitudes have their own $\phi$ dependences, but do not depend on the sign of the charge. Hence the measurement of a cross section asymmetry with respect to the beam charge $\left(d \sigma^{+}-d \sigma^{-}\right) /\left(d \sigma^{+}+d \sigma^{-}\right)$is a way to single out the interference term [11] in the numerator, while the denominator is dominated by a $\phi$-independent $\mathrm{BH}$ contribution. See Ref. [12] for details and full equations.

The photon-helicity-conserving amplitude

$$
\tilde{\mathcal{M}}^{1,1}=F_{1} \mathcal{H}+\frac{x_{B}}{2-x_{B}}\left(F_{1}+F_{2}\right) \tilde{\mathcal{H}}-\frac{t}{4 M_{p}^{2}} F_{2} \mathcal{E}
$$

is given by a linear combination of the Compton form factors (CFFs) $\mathcal{H}, \tilde{\mathcal{H}}$, and $\mathcal{E}$, together with the Dirac and Pauli form factors $F_{1}$ and $F_{2}$ [12]. Here $M_{p}$ denotes the proton mass. The CFFs are convolutions of the corresponding twist-2 GPDs $H, \tilde{\mathcal{H}}$, and $E$ with the hard scattering amplitude.

The $\sin \phi$ modulation accessing the imaginary part of $\tilde{\mathcal{M}}^{1,1}$ has already been observed in cross section asymmetries with respect to the beam helicity $[13,14]$. This paper reports the first measurement of an asymmetry with respect to the beam charge, accessing the real part of $\tilde{\mathcal{M}}^{1,1}$ via a $\cos \phi$ modulation.

Data with an unpolarized hydrogen target were accumulated using the HERMES spectrometer [15] and the longitudinally polarized $27.6 \mathrm{GeV}$ electron and positron beams of the HERA accelerator at DESY. Events were selected if they contained exactly one photon and one charged track identified as the scattered lepton. The hadron contamination in this lepton sample was kept below $1 \%$ by combining the information from a transition-radiation detector, a pre- 
shower scintillator detector, and an electromagnetic calorimeter. The kinematic requirements imposed were $1 \mathrm{GeV}^{2}<Q^{2}<10 \mathrm{GeV}^{2}, \quad 0.03<x_{B}<0.35, \quad W>$ $3 \mathrm{GeV}$, and $\nu<22 \mathrm{GeV}$, where $W$ denotes the initial photon-nucleon invariant mass and $\nu$ is the virtual-photon energy in the target rest frame. The real photon was identified by detecting an energy deposition above $5 \mathrm{GeV}$ in the calorimeter in addition to a signal in the preshower detector, without an associated charged track in the back region of the spectrometer. Unlike in a preliminary stage of this analysis [16], the useful range of the polar angle $\theta_{\gamma^{*} \gamma}$ between the virtual and real photons is not limited by the photon position resolution, due to an improved reconstruction algorithm. Now the restriction on $\theta_{\gamma^{*} \gamma}$ is relaxed to $\theta_{\gamma^{*} \gamma}>5 \mathrm{mrad}$, limited mainly by the electron momentum resolution. In addition, a stricter upper limit of $\theta_{\gamma^{*} \gamma}<$ $45 \mathrm{mrad}$ is imposed in order to improve the signal-tobackground ratio [17].

The recoiling proton was not detected. Hence exclusive events are selected by requiring the missing mass $M_{X}$ of the reaction $e p \rightarrow e \gamma X$ to be close to the proton mass. Figure 1 shows the distribution in $M_{X}^{2}=\left(q+p-q^{\prime}\right)^{2}$, with $q, p$, and $q^{\prime}$ being the four-momenta of the virtual photon, the target nucleon in the initial state, and the real photon, respectively. Mainly due to the resolution in photon energy, the exclusive peak extends to negative values, in which case $M_{X}$ is defined as $-\sqrt{-M_{X}^{2}}$. The exclusive region is defined as $(-1.5 \mathrm{GeV})^{2}<M_{X}^{2}<(1.7 \mathrm{GeV})^{2}$, based on the result of a Monte Carlo simulation (MC), shown in the same figure. The Mo and Tsai formalism [18] is used to simulate the elastic $\mathrm{BH}$ process leaving the target nucleon intact, and the associated $\mathrm{BH}$ process, where the nucleon is excited to a resonant state. For the latter a cross section parametrization for the resonance region is used [19]. Not included in the simulation is the DVCS contribution, which in this kinematic regime is expected to be much smaller than that of the $\mathrm{BH}$ process [20]. The simulation also takes into account the semi-inclusive production of neutral mesons (mostly $\pi^{0}$ ), where all but one of the decay photons escape detection. For this, the MC generator LEPTO [21] in conjunction with a special JETSET [22] fragmentation tune is used, the latter being optimized for energies relevant to HERMES [23]. Not shown in Fig. 1 is the contribution of exclusive $\pi^{0}$ production, which contributes less than $2.5 \%$ to the exclusive region based on the model in Ref. [24]. As shown in Fig. 1, data and MC are in good agreement taking into account that they are both absolutely normalized, and that the MC does not include radiative corrections to the $\mathrm{BH}$ cross section. The $\mathrm{MC}$ yield exceeds the data by about $20 \%$ in the exclusive region, as may be expected [25]. A simulation including second order radiative processes should give an improved MC-data comparison in the full missing-mass region, since a part of the exclusive events experiencing second order radiative corrections will not be lost but reconstructed in the nonexclusive region.

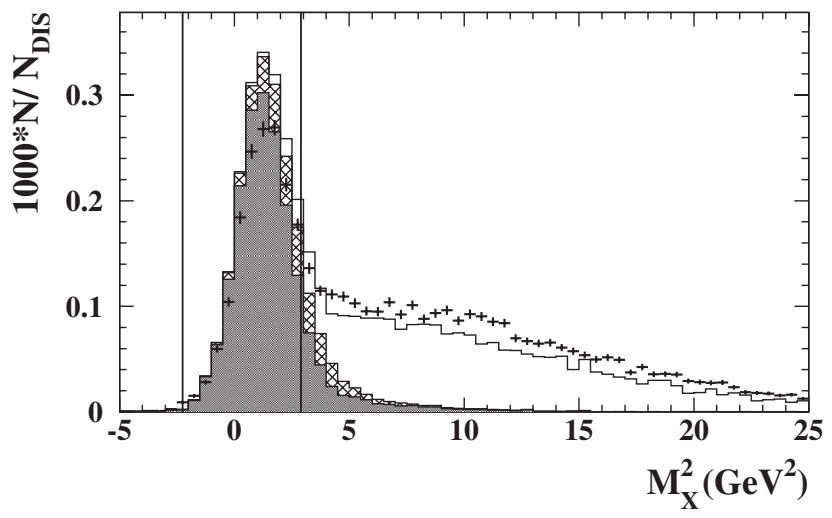

FIG. 1. Distributions in missing-mass squared from data (statistical error bars) and from Monte Carlo simulations (line). The latter include elastic BH (filled area) and associated BH (hatched area) processes as well as semi-inclusive background. The vertical lines enclose the selected exclusive region.

The beam-charge asymmetry is evaluated as

$$
A_{C}(\phi)=\frac{N^{+}(\phi)-N^{-}(\phi)}{N^{+}(\phi)+N^{-}(\phi)}
$$

where $N^{+}(\phi)$ and $N^{-}(\phi)$ represent the single-photon yields per $\phi$ bin, normalized to the number of detected inclusive DIS events using the positron and electron beam, respectively. Since these beams were polarized, sinusoidal contributions appear in the numerator and denominator of Eq. (4), with the last term in Eq. (2) giving the biggest contribution. In order to cancel these contributions, the "symmetrized" beam-charge asymmetry is calculated by replacing $\phi$ by $|\phi|$ in Eq. (4). The result for the exclusive region is displayed in Fig. 2. The shown four-parameter fit yields a nonzero $\cos \phi$ amplitude of $0.060 \pm 0.027$. The result after the background correction described below is given in Table I, last row. The constant term as well as the $\cos 2 \phi$ and $\cos 3 \phi$ terms are compatible with zero. Figure 3 shows the $\cos \phi$ amplitude in several $M_{X}$ bins. At higher $M_{X}$ the result is compatible with zero, confirming the absence of charge-dependent instrumental effects.

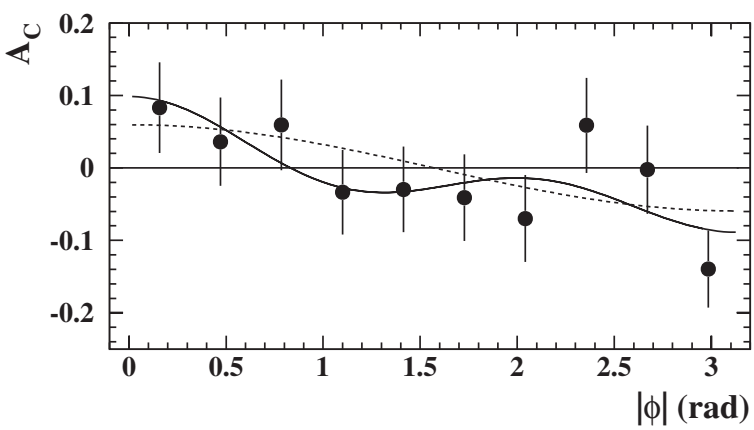

FIG. 2. Beam-charge asymmetry $A_{C}$ for the hard electroproduction of photons off protons as a function of the azimuthal angle $|\phi|$, for the exclusive sample before background correction. Statistical uncertainties are shown. The solid curve represents the four-parameter fit: $(-0.011 \pm 0.019)+$ $(0.060 \pm 0.027) \cos \phi+(0.016 \pm 0.026) \cos 2 \phi+(0.034 \pm$ $0.027) \cos 3 \phi$. The dashed line shows the pure $\cos \phi$ dependence. 


\section{A. AIRAPETIAN et al.}

TABLE I. The $\cos \phi$ amplitude of the beam-charge asymmetry per kinematic bin in $-t$ after background correction and the respective average kinematic values.

\begin{tabular}{l|ccc|c}
\hline \hline$-t$ bin $\left(\mathrm{GeV}^{2}\right)$ & $\langle-t\rangle\left(\mathrm{GeV}^{2}\right)$ & $\left\langle x_{B}\right\rangle$ & $\left\langle Q^{2}\right\rangle\left(\mathrm{GeV}^{2}\right)$ & $A_{C}^{\text {cos } \phi} \pm$ stat. \pm sys. \\
\hline$<0.06$ & 0.03 & 0.08 & 2.0 & $0.024 \pm 0.043 \pm 0.022$ \\
$0.06-0.14$ & 0.09 & 0.10 & 2.6 & $0.020 \pm 0.054 \pm 0.022$ \\
$0.14-0.30$ & 0.20 & 0.11 & 3.0 & $0.071 \pm 0.066 \pm 0.028$ \\
$0.30-0.70$ & 0.42 & 0.12 & 3.7 & $0.377 \pm 0.110 \pm 0.081$ \\
$<0.70$ & 0.12 & 0.10 & 2.5 & $0.063 \pm 0.029 \pm 0.028$ \\
\hline \hline
\end{tabular}

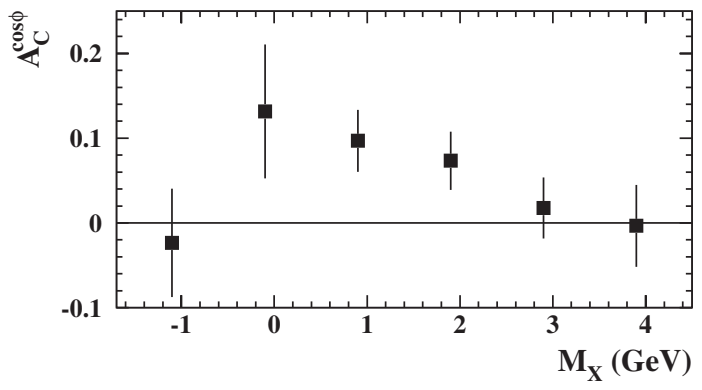

FIG. 3. The $\cos \phi$ amplitude of the beam-charge asymmetry as a function of the missing mass, before background correction. Statistical uncertainties are shown.

As the recoiling proton remains undetected, $t$ is inferred from the measurement of the other final-state particles. For elastic events, kinematics relate the energy with the direction of the real photon, opening the possibility to omit the real-photon energy, which is the quantity subject to larger uncertainty. Thus the value of $t$ in the exclusive region is calculated as

$$
t=\frac{-Q^{2}-2 \nu\left(\nu-\sqrt{\nu^{2}+Q^{2}} \cos \theta_{\gamma^{*} \gamma}\right)}{1+\frac{1}{M_{p}}\left(\nu-\sqrt{\nu^{2}+Q^{2}} \cos \theta_{\gamma^{*} \gamma}\right)} .
$$

The error caused by applying this expression to inelastic events ( $\approx 17 \%$ in the exclusive region) is accounted for in the MC simulation that is used to calculate the fractional contribution of background processes per kinematic bin in $-t$ (see Ref. [17] for details).

Figure 4 shows the $\cos \phi$ amplitude derived from the four-parameter fit in each of four bins in $-t$. In each bin, this result is corrected for the semi-inclusive background, which is treated as a dilution since the background asymmetry can only be nonzero at next-to-leading order in QED. The total background contribution is about $6 \%$ as derived from the MC simulation, wherein the elastic and associated BH contributions are scaled down by the $20 \%$ described above. The resulting $\cos \phi$ amplitudes are expected to originate from only elastic and associated production. The associated $\mathrm{BH}$ processes contribute about $5 \%$, $11 \%, 18 \%$, and $29 \%$ to the yields in the four $-t$ bins, or $11 \%$ in the full $t$-range, with an estimated fractional uncertainty of $10 \%$. The dominant contribution to the total systematic uncertainty of the $\cos \phi$ amplitudes stems from effects due to possible deviations of the detector and/or the beam from their nominal positions. These effects can be as
PHYSICAL REVIEW D 75, 011103(R) (2007)

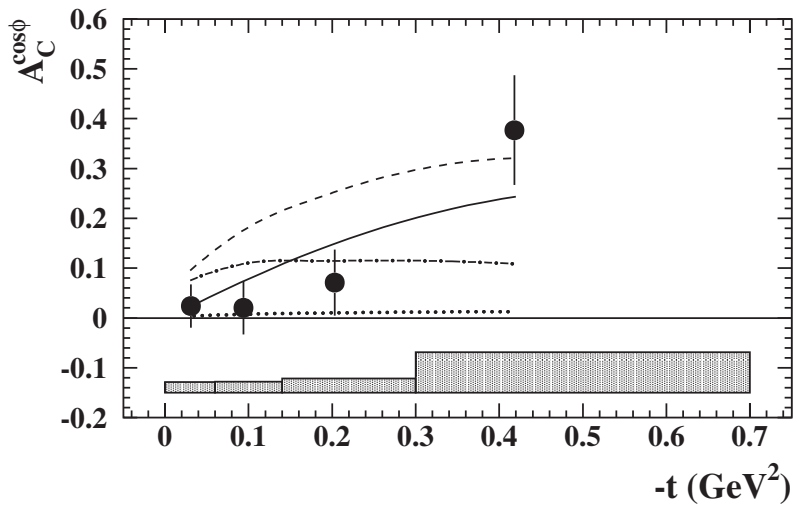

FIG. 4. The $\cos \phi$ amplitude of the beam-charge asymmetry as a function of $-t$ for the exclusive region $\left(-1.5 \mathrm{GeV}<M_{X}<\right.$ $1.7 \mathrm{GeV}$ ), after background correction. The error bars (band) represent(s) the statistical (systematic) uncertainties. The calculations based on GPD models [24,27] use either a factorized $t$-dependence with (dashed-dotted line) or without (dotted line) the $D$-term contribution, or a Regge-inspired $t$-dependence with (dashed line) or without (solid line) the $D$-term contribution.

large as 0.02 per bin. Based on the models in Ref. [20], acceptance and smearing effects can contribute up to $20 \%$ of the $\cos \phi$ amplitude, and thus dominate the systematic uncertainty in the last $-t$ bin. The other sources of systematic uncertainties are due to a possible difference in the calorimeter calibration between the two data sets, the uncertainties from the semi-inclusive background correction described above, and the dilution of the asymmetry due to exclusively produced $\pi^{0}$ mesons misidentified as photons. These contributions are combined quadratically in the total systematic uncertainty per bin in $-t$, given in Table I. ${ }^{1}$ Not included is any contribution due to additional QED vertices, as the most significant of these has been estimated to be negligible, at least in the case of polarization asymmetries [26].

The theoretical calculations for the $e p \rightarrow e p \gamma$ process shown in Fig. 4 employ GPD models developed in Refs. [24,27], which are based on the widely used framework of double distributions [28]. The model parameters of interest are those that change the GPD $H$ since the impact of the GPDs $\tilde{H}$ and $E$ is suppressed at small values of $x_{B}$ and $-t$, respectively (cf. Eq. (3)). The code of Ref. [29] was used to calculate the values for the $\cos \phi$ amplitude of the beam-charge asymmetry at the average kinematics (see Table I) of every $-t$ bin and not at the kinematics of every event since it is too computationally intensive. The difference between these two approaches is strongly model dependent: Tests [30] show differences of up to $20 \%$ using the models in Ref. [20], which are equivalent to the fac-

\footnotetext{
${ }^{1}$ Note that a preliminary result of this analysis [16] with a $t$-averaged value of $0.11 \pm 0.04$ (stat.) \pm 0.03 (sys.) was derived at a much larger mean $-t$ value $\left(\langle-t\rangle=0.27 \mathrm{GeV}^{2}\right)$ due to different requirements on $\theta_{\gamma^{*} \gamma}$, as described above. It thus cannot be compared to the $t$-averaged result given in Table I but approximately to the result in the third $-t$ bin.
} 
torized models in Fig. 4. Four different parameter sets are selected by choosing either a factorized or a Reggeinspired $t$-dependence, each with or without the contribution of a negative value of the so-called $D$-term [31], which is related to the spontaneous breaking of chiral symmetry in QCD [27]. It contributes to the real part of the DVCS amplitude only and therefore can be investigated with the beam-charge asymmetry for the first time. A large positive $D$-term is ruled out by these data [16], since it leads to a negative value for the beam-charge asymmetry. The parameters $b_{\text {val }}$ and $b_{\text {sea }}$ in the profile function [32] are fixed at unity, since the beam-charge asymmetry has been shown to be largely insensitive to them [17]. In comparing the predictions to the data at large $-t$, it should be borne in mind that the model calculations do not include associated production, which increases with $-t$ as mentioned above. The three data points at small $-t$ exclude the model based on the Regge-inspired $t$-dependence with the $D$-term contribution.

These data are in agreement with a very recent calculation based on a dual parametrization of GPDs and either of two models for the $t$-dependence [33]. Like the calculations shown in Fig. 4, these calculations were performed at the average kinematics (see Table I) of every $-t$ bin. Earlier model calculations were mostly done not as a function of $t$ but at the average kinematics of the preliminary result [16], and thus cannot rigorously be compared to this measurement, see, e.g., Refs. [7,12,34,35]. However, since they span a wider or different range for the magnitude of the beam-charge asymmetry when compared to the models shown here, it is apparent that this measurement can constrain the GPD $H$.

In conclusion, a beam-charge azimuthal asymmetry in electroproduction of real photons has been measured for the first time. A $\cos \phi$ dependence has been observed in a kinematic region where the target proton is predominantly left intact. The $\cos \phi$ dependence is predicted to arise from the interference between the deeply virtual Compton scattering and Bethe-Heitler processes. These data can already be used to distinguish among theoretical models for generalized parton distributions.

We gratefully acknowledge the DESY management for its support, the staff at DESY and the collaborating institutions for their significant effort, and our national funding agencies for financial support.
[1] D. Müller et al., Fortschr. Phys. 42, 101 (1994).

[2] X. Ji, Phys. Rev. Lett. 78, 610 (1997); Phys. Rev. D 55, 7114 (1997).

[3] A. V. Radyushkin, Phys. Rev. D 56, 5524 (1997).

[4] M. Burkardt, Phys. Rev. D 62, 071503 (2000); 66, 119903(E) (2002).

[5] M. Diehl, Eur. Phys. J. C 25, 223 (2002); 31, 277(E) (2003).

[6] J. P. Ralston and B. Pire, Phys. Rev. D 66, 111501 (2002).

[7] A. V. Belitsky and D. Müller, Nucl. Phys. A711, 118 (2002).

[8] M. Burkardt, Int. J. Mod. Phys. A 18, 173 (2003).

[9] A. Bacchetta et al., Phys. Rev. D 70, 117504 (2004).

[10] M. Diehl et al., Phys. Lett. B 411, 193 (1997).

[11] S. J. Brodsky, F. E. Close, and J. F. Gunion, Phys. Rev. D 6, 177 (1972).

[12] A. V. Belitsky, D. Müller, and A. Kirchner, Nucl. Phys. B629, 323 (2002).

[13] A. Airapetian et al. (HERMES Collaboration), Phys. Rev. Lett. 87, 182001 (2001).

[14] S. Stepanyan et al. (CLAS Collaboration), Phys. Rev. Lett. 87, 182002 (2001).

[15] K. Ackerstaff et al. (HERMES Collaboration), Nucl. Instrum. Methods Phys. Res., Sect. A 417, 230 (1998).

[16] F. Ellinghaus (HERMES Collaboration), Nucl. Phys. A711, 171 (2002).

[17] F. Ellinghaus, Ph.D. thesis, Humboldt University Berlin, Germany [DESY Report No. 2004-005 2004 (unpublished)].

[18] L. W. Mo and Y. S. Tsai, Rev. Mod. Phys. 41, 205 (1969).

[19] F. W. Brasse et al., Nucl. Phys. B110, 413 (1976).

[20] V. A. Korotkov and W.-D. Nowak, Eur. Phys. J. C 23, 455
(2002).

[21] G. Ingelman, A. Edin, and J. Rathsman, Comput. Phys. Commun. 101, 108 (1997).

[22] T. Sjöstrand, Comput. Phys. Commun. 82, 74 (1994).

[23] A. Hillenbrand, Ph.D. thesis, University ErlangenNuremberg, Germany [DESY Report No. 2005-035 2005 (unpublished)].

[24] M. Vanderhaeghen, P. A. M. Guichon, and M. Guidal, Phys. Rev. D 60, 094017 (1999).

[25] M. Vanderhaeghen et al., Phys. Rev. C 62, 025501 (2000).

[26] A. V. Afanasev, M. I. Konchatnij, and N.P. Merenkov, J. Exp. Theor. Phys. 102, 220 (2006).

[27] K. Goeke, M. V. Polyakov, and M. Vanderhaeghen, Prog. Part. Nucl. Phys. 47, 401 (2001).

[28] A. V. Radyushkin, Phys. Rev. D 59, 014030 (1998); Phys. Lett. B 449, 81 (1999).

[29] M. Vanderhaeghen, P. A. M. Guichon, and M. Guidal (Private Communication).

[30] B. Krauss, Ph.D. thesis, University Erlangen-Nuremberg, Germany [DESY Report No. 2005-008 2005 (unpublished)].

[31] M. V. Polyakov and C. Weiss, Phys. Rev. D 60, 114017 (1999).

[32] I. V. Musatov and A. V. Radyushkin, Phys. Rev. D 61, 074027 (2000).

[33] V. Guzey and T. Teckentrup, Phys. Rev. D 74, 054027 (2006).

[34] N. Kivel, M. V. Polyakov, and M. Vanderhaeghen, Phys. Rev. D 63, 114014 (2001).

[35] A. Freund, M. McDermott, and M. Strikman, Phys. Rev. D 67, 036001 (2003). 